\title{
Outcomes in Patients with SARS-CoV-2 and Clostridioides difficile Coinfection
}

\author{
Kanika Sehgal (1D) \\ Hind J Fadel ${ }^{2}$ \\ Aaron J Tande ${ }^{2}$ \\ Darrell S Pardi ${ }^{\prime}$ \\ Sahil Khanna (iD) \\ 'Division of Gastroenterology and \\ Hepatology, Mayo Clinic, Rochester, MN, \\ USA; ${ }^{2}$ Division of Infectious Diseases, \\ Mayo Clinic, Rochester, MN, USA
}

\begin{abstract}
Background: Coronavirus infectious disease 2019 (COVID-19) is primarily a respiratory disease. However, it may manifest with gastrointestinal symptoms that may overlap with Clostridioides difficile infection (CDI). COVID-19 appears to have higher mortality in those with comorbidities. We aimed to assess the outcomes of coinfection in these patients.

Methods: A retrospective chart review was conducted to identify patients with CDI and COVID-19 from January 1st, 2020 to November 17th, 2020. Both infections were diagnosed via PCR. Clinical features, treatment for COVID-19 and CDI and outcomes including intensive care unit admission, colectomy, 30 day-mortality and long-term complications were analyzed.
\end{abstract}

Results: Overall, 21 patients (20 hospitalized) with median age 70.9 years (range 51.8-90.7 years) had CDI and COVID-19 within 4 weeks of each other. Of these, 4 patients (19\%) with CDI were diagnosed with COVID-19 at the time of admission, 12 (57\%) had CDI diagnosed after COVID-19, and 5 (23.9\%) developed COVID-19 within 4 weeks after CDI. Fourteen patients $(66.7 \%)$ were treated with medications directed against COVID-19 including remdesivir and dexamethasone $(n=7)$, remdesivir with convalescent plasma $(n=1)$, remdesivir $(n=5)$ and dexamethasone $(n=1)$. The most common treatment for CDI was oral vancomycin in 20 patients $(95.2 \%)$, and 1 patient received intravenous metronidazole. No patient required colectomy for CDI but $2(9.5 \%)$ required ICU admission. Four patients (19\%) died likely due to COVID-19 with median age 80 years (range 61-90 years).

Conclusion: The relationship between COVID-19 and CDI is poorly understood, and studies are required to further investigate this association. Whether coinfection results in a worsening of outcomes, including mortality and clinical course, are questions that should be answered in future research studies. Diagnosing both infections for appropriate management is vital in light of overlapping symptoms.

Keywords: diarrhea, infection, C. difficile, microbiome, antibiotics, COVID-19, mortality

\section{Introduction}

Coronavirus infectious disease 2019 (COVID-19), caused by the severe acute respiratory syndrome coronavirus 2 (SARS-CoV-2) is primarily considered to be a respiratory illness with most common symptoms being fever, fatigue, cough and dyspnea. ${ }^{1}$ Mortality from COVID-19 is variable and higher in those with systemic comorbidities. COVID-19 also presents with gastrointestinal symptoms such as diarrhea, loss of appetite and nausea. ${ }^{1,2}$ Patients with COVID-19 have been managed with antimicrobials. $^{3,4}$ A common side effect of these antimicrobials is diarrhea and $C$. difficile infection (CDI). ${ }^{5}$ Additionally, it has been discovered that bacterial coinfections can occur when the coronavirus is being eradicated from the patients' lungs as
Correspondence: Sahil Khanna Division of Gastroenterology and Hepatology, Mayo Clinic, 200 First St SW Rochester, MN, 55905, USA

Tel + I 507-266-4347

$\mathrm{Fax}+1$ 507-293-3917

Email Khanna.sahil@mayo.edu 
the adaptive immune reaction towards the virus fails the reaction of innate immunity against bacterial infection. ${ }^{6}$

The SARS-CoV-2 utilizes the angiotensin converting enzyme-2 (ACE-2) receptor for cellular entry, which is expressed on colonocytes. ${ }^{7}$ COVID-19 has been associated with gut microbial dysbiosis, ${ }^{8}$ impairing colonization resistance and an increased risk of CDI. ${ }^{9}$ Coinfection with these pathogens can lead to poor outcomes. A study of 9 patients with concomitant CDI and COVID-19 demonstrated a $44 \%$ mortality. ${ }^{10}$ Due to overlapping symptoms and high rates of complications, it is important to manage these coinfected patients aggressively.

\section{Methods}

We conducted a retrospective study of patients with CDI and COVID-19 from March 1st, 2020 to November 17th 2020, to delineate management and outcomes in these patients. Data abstracted included demographics, symptoms, risk factors for CDI and the temporal association between COVID19 and CDI. In all patients, COVID-19 was diagnosed by a swab from the nasopharynx via PCR and CDI was diagnosed using toxin detection in the stool via PCR. Treatment for COVID-19 and CDI and outcomes including ICU admission, colectomy and 30-day mortality were recorded. The research is approved by the Mayo Clinic Institutional Review Board and informed consent was not required since this was a minimal risk retrospective study.

\section{Results}

We identified 21 patients (20 hospitalized) with median age 70.9 years (range 51.8-90.7) who had CDI and COVID-19 within 4 weeks of each other (Figure 1), 12 $(57 \%)$ of whom were female. Except 2 patients, all others had a medical history of comorbidities, including hypertension in 13 patients (68.4\%), followed by dyslipidemia in 9 patients $(47.3 \%)$, type 2 diabetes mellitus in 5 $(26.3 \%)$, neoplasias in $5(26.3 \%)$ (such as adenomatous colon polyps, glioblastoma multiforme, prostate cancer, squamous cell cancer and multiple myeloma), hypothyroidism in $4(21 \%)$ and others included coronary artery disease, chronic obstructive pulmonary disease and type 1 diabetes mellitus. Four patients (19\%) with CDI were diagnosed with COVID-19 at the time of admission, 12 (57\%) had CDI diagnosed after COVID-19 (median 2.4

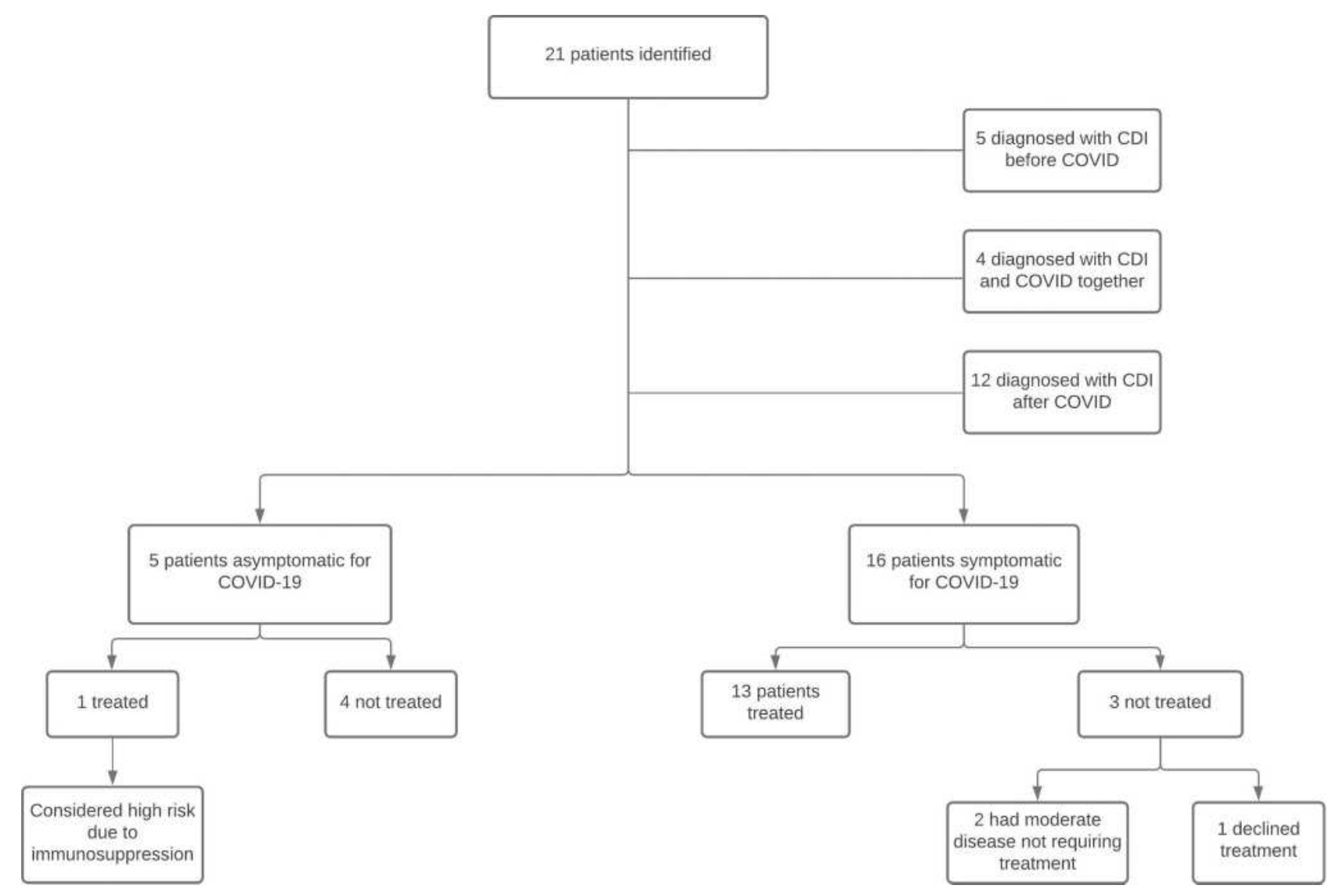

Figure I Patients identified with CDI and COVID-19 within 4 weeks of each other, with their symptoms and eventual treatment status. 
days, range 1.2-17) and 5 (23.9\%) developed COVID-19 within 4 weeks after CDI (median 9 days, range 0.5-17.3).

Five patients (23.9\%) were considered asymptomatic for COVID-19 (absence of respiratory symptoms or fever), while the rest had symptoms including fever, cough, dyspnea and shortness of breath. Of the asymptomatic patients, 3 were screened as a part of routine testing, 1 was screened for liver transplantation status and 1 was tested due to infiltrates seen on chest $\mathrm{x}$-ray. COVID-19 asymptomatic patients were hospitalized for urinary tract infection, generalized weakness, cellulitis, hematochezia and diarrhea or liver transplant complications, all deemed unrelated to COVID-19. One of the five asymptomatic patients was eventually treated for COVID-19 with remdesivir in conjunction with convalescent plasma owing to the patient's immunosuppressed status to prevent complications.

Of the 21 patients, 7 (33.3\%) were not treated with COVID-19 directed therapies. Of these, 6 were managed supportively (4 were asymptomatic and 2 had moderate illness according to NIH guidelines) and 1 declined treatment. Fourteen patients (66.7\%) had COVID-19 targeted treatment. A combination of remdesivir and dexamethasone was used in 7 patients, remdesivir with convalescent plasma in 1 patient, remdesivir alone in 5 patients and dexamethasone alone in 1 patient.

Overall, 16 of 21 patients (76.1\%) were exposed to antibiotics prior to CDI and $8(38 \%)$ were on immunosuppression. Seven patients (33\%) had other coinfections at the time of CDI including Campylobacter jejuni, UTI with Enterobacter, diverticulitis, enteropathogenic Escherichia coli and opportunistic infections such as Cytomegalovirus viremia, Pseudomonas aeruginosa and Cryptococcus neoformans infection. The median duration of concomitant antibacterial therapy given was 7 days (range 3-9 days). The most common treatment for CDI was oral vancomycin in 20 patients $(95.2 \%)$, out of which one was transitioned to oral fidaxomicin and the remaining 1 patient received intravenous metronidazole. No patient required colectomy for CDI. All patients receiving treatment for CDI eventually resolved with standard vancomycin treatment regimens, except 6 patients, who needed a longer course of vancomycin therapy to achieve resolution.

Two patients $(9.5 \%)$ required Intensive Care Unit admission in the setting of worsening status due to COVID-19 and 4 patients (median age 80 years, range 61-90) died within 30 days. The deaths were related to COVID-19, and these patients had multiple comorbidities including hypertension, hyperlipidemia, hypothyroidism, acute and chronic kidney injury, chronic obstructive pulmonary disease, glioblastoma multiforme and Alzheimer's disease. Of the 4 deaths, 2 had an immunosuppressed state and 1 had a urinary tract infection with multidrug resistant Enterobacter as well as soft tissue infection with methicillin-resistant Staphylococcus aureus.

\section{Discussion}

The relationship between CDI and COVID-19 is poorly understood, and more data are needed to understand the relationship between the two infections. Whether coinfection results in worsening mortality or more complications remains unanswered. In our dataset, most patients have had a prior history of antibiotic usage that could be potentially implicated in causing CDI. Of patients with both COVID-19 and CDI, none required colectomy and 2 needed Intensive Care Unit admission. All observed deaths were related to COVID-19 in the elderly with comorbidities and the observed mortality rate with coinfection was $19 \%$. A limitation of our study is the small sample size. Due to the retrospective nature of this study, determining the time to resolution of diarrhea in these patients was not possible. Additionally, it was not feasible to gather information about diarrheal symptoms in all COVID-19 infected patients during the timeframe of our study as patients are diagnosed with COVID-19 in various clinical care settings, including inpatient, outpatient, drivethrough, urgent care, which made gathering reliable data difficult. Further, information about stool sampling in such COVID-19 positive patients was not feasible either.

In contrast to the previous study, ${ }^{10}$ patients in our study were not treated with antibiotics for COVID-19 and CDI seemed to have developed due to prior antibiotic exposure for other indications. The COVID-19 mortality rate we encountered with coinfection was $19 \%$ (4 out of 21 patients), which is lower than the rate of $44 \%$ seen in the previous study. ${ }^{10}$ However, lower mortality rates have been observed with the Mayo Clinic model of care and research, as reported in a study that compared Mayo Clinic outcomes in COVID-19 patients with the rates reported nationally. ${ }^{11}$ Further, the observed mortality in our study was noted in patients that were older and had comorbidities.

The association between COVID-19 and CDI still needs exploration. Several patients with COVID-19 present with diarrhea, which is often assumed to be COVID-19 related. If such patients undergo CDI PCR testing routinely, we might 
have a more definitive answer of the association in question. In light of potentially overlapping symptoms, assessment for both infections by laboratory diagnosis is pertinent for administering appropriate treatment.

\section{Summary}

COVID-19 manifests with gastrointestinal symptoms similar to Clostridioides difficile infection (CDI), making it pertinent to consider both conditions in a patient with diarrhea. We identified 21 patients with COVID-19 and CDI within 4 weeks of each other. A high 30-day mortality of $19 \%$ was observed, mostly in the elderly.

\section{Disclosure}

The authors report no conflicts of interest in this work.

\section{References}

1. Tariq R, Saha S, Furqan F, Hassett L, Pardi D, Khanna S. Prevalence and mortality of COVID-19 patients with gastrointestinal symptoms: a systematic review and meta-analysis. Mayo Clin Proc. 2020;95 (8):1632-1648. doi:10.1016/j.mayocp.2020.06.003

2. Han C, Duan C, Zhang S, et al. Digestive symptoms in COVID-19 patients with mild disease severity: clinical presentation, stool viral RNA testing, and outcomes. Am $J$ Gastroenterol. 2020;115 (6):916-923. doi:10.14309/ajg.0000000000000664
3. Yang X, Yu Y, Xu J, et al. Clinical course and outcomes of critically ill patients with SARS-CoV-2 pneumonia in Wuhan, China: a single-centered, retrospective, observational study. Lancet Respir Med. 2020;8(5):475-481. doi:10.1016/S2213-2600(20)30079-5

4. Chen T, Wu D, Chen $\mathrm{H}$, et al. Clinical characteristics of 113 deceased patients with coronavirus disease 2019: retrospective study. BMJ. 2020;368:m1091. doi:10.1136/bmj.m1091

5. Saha S, Khanna S. Management of Clostridioides difficile colitis: insights for the gastroenterologist. Therap Adv Gastroenterol. 2019;12:1756284819847651. doi:10.1177/1756284819847651

6. Mirzaei R, Goodarzi P, Asadi M, et al. Bacterial co-infections with SARS-CoV-2. IUBMB Life. 2020;72(10):2097-2111. doi:10.1002/ iub. 2356

7. Li W, Moore MJ, Vasilieva N, et al. Angiotensin-converting enzyme 2 is a functional receptor for the SARS coronavirus. Nature. 2003;426(6965):450-454. doi:10.1038/nature02145

8. Lakkasani S, Chan KH, Shaaban HS. Clostridiodes difficile in COVID-19 patients, Detroit, Michigan, USA, March-April 2020. Emerg Infect Dis. 2020;26(9):2299-2300. doi:10.3201/ eid2609.202505

9. Jacobs NF. Antibiotic-induced diarrhea and pseudomembranous colitis. Postgrad Med. 1994;95(8):111-120. doi:10.1080/ 00325481.1994.11945865

10. Sandhu A, Tillotson G, Polistico J, et al. Clostridioides difficile in COVID-19 patients, Detroit, Michigan, USA, March-April 2020. Emerg Infect Dis. 2020;26(9):2272. doi:10.3201/eid2609.202126

11. O'Horo JC, Cerhan JR, Cahn EJ, et al. Outcomes of COVID-19 with the Mayo Clinic model of care and research. Mayo Clin Proc. 2021;96(3):601-618. doi:10.1016/j.mayocp.2020.12.006
Infection and Drug Resistance

\section{Publish your work in this journal}

Infection and Drug Resistance is an international, peer-reviewed openaccess journal that focuses on the optimal treatment of infection (bacterial, fungal and viral) and the development and institution of preventive strategies to minimize the development and spread of resistance. The journal is specifically concerned with the epidemiology of antibiotic resistance and the mechanisms of resistance development and diffusion in both hospitals and the community. The manuscript management system is completely online and includes a very quick and fair peerreview system, which is all easy to use. Visit http://www.dovepress.com/ testimonials.php to read real quotes from published authors. 